\title{
COMMENTARY
}

\section{Out-of-hospital CPR: better outcome for our patients}

\author{
Enrica Golia",2, Maddalena Piro and Marco Tubaro* \\ See related research by Gräsner et al., http://ccforum.com/content/15/1/R61
}

\begin{abstract}
Out-of-hospital cardiac arrest is a leading cause of death in developed countries and early resuscitation attempts are crucial to improve survival rates and neurological outcome. Gräsner and colleagues performed an intriguing analysis on the combined approach of mild therapeutic hypothermia (MTH) and immediate percutaneous coronary intervention (PCI) for post-resuscitation care of 584 patients with out-ofhospital cardiac arrest from the German Resuscitation Registry. PCl was independently associated with good neurological outcome at hospital discharge after successful resuscitation, and MTH was associated as an independent factor with increased chance of 24-hour survival. Moreover, a binary logistic regression analysis did not show statistical significance for MTH, in addition to $\mathrm{PCl}$, as an independent predictor for good neurological outcome. The present study supports the evidence that post-resuscitation care based on standardized protocols is beneficial after successful resuscitation. Further prospective and randomized studies are warranted to elucidate criteria for a better selection of candidates for those strategies and to evaluate the potential, in terms of neurological outcome at hospital discharge, of a prehospital cooling strategy in patients who cannot be referred to immediate PCl.
\end{abstract}

Gräsner and colleagues, in the previous issue of Critical Care, perform an intriguing analysis on the combined approach of mild therapeutic hypothermia (MTH) and immediate percutaneous coronary intervention (PCI) for post-resuscitation care of patients with out-of-hospital cardiac arrest (OHCA) [1].

*Correspondence: m.tubaro@alice.it

${ }^{2} \mathrm{ICCU}$, Cardiovascular Department, San Filippo Neri Hospital, Via Martinotti 20, 00135 Rome, Italy

Full list of author information is available at the end of the article
OHCA is a leading cause of death in developed countries and early resuscitation attempts through basic and then advanced cardiac life support therapies are crucial to improve survival rates and neurological outcome [2]. The outcome of patients experiencing OHCA remains poor and varies significantly, according to rhythm characteristics (shockable vs. nonshockable) and other clinical features. However, the long-term prognosis of patients discharged alive has improved [3]. Because approximately $70 \%$ of OHCA patients have underlying acute myocardial infarction, several studies focused on the role of reperfusion strategies, such as thrombolysis and primary PCI, during cardiac arrest or, most often, after return of spontaneous circulation [4-7]. In particular, immediate successful PCI is associated with improved hospital survival, in the absence of obvious extracardiac causes for the arrest [4-6]. Primary PCI is effective independently of the electrocardiogram pattern (that is, with or without ST-segment elevation) [4], suggesting a poor predictive value of the electrocardiogram in identifying patients who may benefit from coronary intervention.

Since techniques in resuscitation have improved, the population of patients with anoxic brain injury is growing. The induction of MTH (32 to $34^{\circ} \mathrm{C}$ ) for 24 hours has been shown to improve neurologic outcome of patients successfully resuscitated after cardiac arrest [8-10]. This improvement was demonstrated in two randomized controlled trials in patients with ventricular fibrillation as the initial cardiac rhythm $[8,9]$. Two meta-analyses found that MTH improves short-term neurologic outcome and survival in patients resuscitated from OHCA of presumed cardiac origin, with ventricular fibrillation/ ventricular tachycardia as the initial rhythm $[11,12]$.

The German Resuscitation Registry is a large registry started in 1998 for the collection of preclinical datasets on the attempts of out-of-hospital resuscitation. Since 2004 this database has been extended to the post-resuscitation datasets [1]. Despite obvious limitations, data from largescale registries provide useful insights on the feasibility and safety of new therapeutic strategies, as well as on offlabel indications. These insights are particularly useful in settings such as emergency care in which randomized 
controlled trials are challenging, primarily because of ethical concerns. Gräsner and colleagues evaluated 584 patients from the German Resuscitation Registry with complete pre-resuscitation and post-resuscitation datasets [1]. The authors described the effects of a combined approach for the early treatment of OHCA patients with or without a shockable rhythm. They found that both PCI and MTH were associated with good neurological outcome and increased survival at 24 hours after return of spontaneous circulation, in line with previous reports [4-6]. The authors evaluated the effects of both MTH and PCI used alone and in combination: they found a beneficial effect even for PCI alone, while in a large previous report immediate PCI after OHCA was associated with $\mathrm{MTH}$ in $85 \%$ of patients [4]. Interestingly, data from binary logistic regression analysis showed that $\mathrm{MTH}$ was not an independent predictor of good neurologic recovery, when used in addition to PCI [1].

Some details are lacking in the present study, however, such as timing and the devices used to achieve cooling, as well as PCI details. Moreover, data on the potential advantage of the combination of $\mathrm{PCI}$ and $\mathrm{MTH}$ versus PCI alone or MTH alone are lacking.

In the PRINCE study, induction of hypothermia with intranasal cooling devices in a prehospital setting may be useful in reducing time intervals required to cool the patients [13]. An outcome difference in comparison with in-hospital cooling was not found; a post-hoc analysis of patients in which cardiopulmonary resuscitation (CPR) was started within 10 minutes demonstrated a significant effect of prehospital cooling on survival rate and neurological recovery. The potential of this approach in patients not immediately undergoing PCI due to lack of catheterization facilities is worth studying.

Some other interesting factors deserve further attention, particularly the association of fibrinolysis with a worse outcome. This association is in contrast to a previous report by Böttiger and colleagues, who found no difference of outcome in comparison with placebo using thrombolysis during resuscitation [7], without adjunctive antithrombotic therapies. Fibrinolysis is usually considered relatively contraindicated after prolonged CPR, because of the increased risk of bleeding. It could be useful to establish whether factors such as the timing of fibrinolysis administration, the duration of CPR and adjunctive antithrombotic therapies could have influenced the unfavorable effects observed in the present study.

Post-resuscitation care based on more standardized protocols including PCI and MTH may be beneficial after successful resuscitation. Further studies are warranted to elucidate criteria for a better selection of candidates, and to demonstrate whether a prehospital rapid cooling strategy during early CPR may be independently associated with an improvement of neurological outcome in patients who cannot be referred for immediate coronary angiography.

\section{Abbreviations}

CPR, cardiopulmonary resuscitation; MTH, mild therapeutic hypothermia; $\mathrm{OHCA}$, out-of-hospital cardiac arrest; $\mathrm{PCl}$, percutaneous coronary intervention.

\section{Author details}

'Division of Cardiology, Second University of Naples, Monaldi Hospital, Via L. Bianchi, 80131, Naples, Italy. ${ }^{2} \mathrm{CCCU}$, Cardiovascular Department, San Filippo Neri Hospital, Via Martinotti 20, 00135 Rome, Italy.

\section{Competing interests}

The authors declare that they have no competing interests.

Published: 7 April 2011

\section{References}

1. Gräsner JT, Meybohm P, Caliebe A, Bottiger BW, Wnent J, Messelken M, Jantzen T, Zeng T, Strickmann B, Bohn A, Fischer H, Scholz J, Fischer M; German Resuscitation Registry Study Group: Postresuscitation care with mild therapeutic hypothermia and coronary intervention after out-ofhospital cardiopulmonary resuscitation: a prospective registry analysis. Crit Care 2011, 15:R61.

2. Bunch TJ, White RD, Gersh BJ, Meverden RA, Hodge DO, Ballman KV, Hammill SC, Shen WK, Packer DL: Long-term outcomes of out-of-hospital cardiac arrest after successful early defibrillation. N Engl J Med 2003, 348:2626-2633.

3. Rea TD, Crouthamel M, Eisenberg MS, Becker L, Lima AR: Temporal patterns in long-term survival after resuscitation from out-of-hospital cardiac arrest. Circulation 2003, 108:1196-1201.

4. Dumas F, Cariou A, Manzo-Silberman S, Grimaldi D, Vivien B, Rosencher J, Empana JP, Carli P, Mira JP, Jouven X, Spaulding C: Immediate percutaneous coronary intervention is associated with better survival after out-ofhospital cardiac arrest: insights from the PROCAT (Parisian Region Out of hospital Cardiac ArresT) registry. Circ Cardiovasc Interv 2010, 3:200-207.

5. Spaulding CM, Joly LM, Rosenberg A, Monchi M, Weber SN, Dhainaut JF, Carli $\mathrm{P}$ : Immediate coronary angiography in survivors of out-of-hospital cardiac arrest. N Engl J Med 1997, 336:1629-1633.

6. Reynolds JC, Callaway CW, El Khoudary SR, Moore CG, Alvarez RJ, Rittenberger $\mathrm{JC}$ : Coronary angiography predicts improved outcome following cardiac arrest: propensity-adjusted analysis. J Intensive Care Med 2009, 24:179-186.

7. Böttiger BW, Arntz HR, Chamberlain DA, Bluhmki E, Belmans A, Danays T, Carli PA, Adgey JA, Bode C, Wenzel V; TROICA Trial Investigators and European Resuscitation Council Study Group: Thrombolysis during resuscitation for out-of-hospital cardiac arrest. N Eng/ J Med 2008, 359:2651-2662.

8. Hypothermia after Cardiac Arrest Study Group: Mild therapeutic hypothermia to improve the neurologic outcome after cardiac arrest. N Engl J Med 2002, 346:549-556.

9. Bernard SA, Gray TW, Buist MD, Jones BM, Silvester W, Gutteridge G, Smith K: Treatment of comatose survivors of out-of-hospital cardiac arrest with induced hypothermia. N Engl J Med 2002, 346:557-563.

10. Felberg, RA, Krieger, DW, Chuang, R, Persse DE, Burgin WS, Hickenbottom SL, Morgenstern LB, Rosales O, Grotta JC: Hypothermia after cardiac arrest: feasibility and safety of an external cooling protocol. Circulation 2001, 104:1799-1804.

11. Arrich, J, Holzer, M, Herkner, H, Müllner, M: Hypothermia for neuroprotection in adults after cardiopulmonary resuscitation. Cochrane Database System Rev 2009, 4:CD004128. doi: 10.1002/14651858.CD004128.pub2.

12. Cheung, KW, Green, RS, Magee, KD: Systematic review of randomized controlled trials of therapeutic hypothermia as a neuroprotectant in post cardiac arrest patients. CJEM 2006, 8:329-337.

13. Castrén M, Nordberg P, Svensson L, Taccone F, Vincent JL, Desruelles D, Eichwede F, Mols P, Schwab T, Vergnion M, Storm C, Pesenti A, Pachl J, Guérisse F, Elste T, Roessler M, Fritz H, Durnez P, Busch HJ, Inderbitzen B, Barbut D: Intra-arrest transnasal evaporative cooling: a randomized, prehospital, multicenter study (PRINCE: Pre-ROSC IntraNasal Cooling Effectiveness). Circulation 2010, 122:729-736.

doi:10.1186/cc10108

Cite this article as: Golia E, et al:: Out-of-hospital CPR: better outcome for our patients. Critical Care 2011, 15:149. 